\title{
Development and Characterization of Ternary Hybrid Membranes in Natural Polymers and their Application in the Microbiology Field
}

Bouhadiba $\mathbf{K}^{1}$, Djennad $\mathbf{M H}^{1}$ and Hammadi $\mathbf{K}^{\mathbf{2}^{*}}$

${ }^{1}$ Laboratory of SEA2M, Department of Chemistry, Faculty of Sciences and Technology, University of Mostaganem, Mostaganem, Algeria

${ }^{2}$ Pedagogical Laboratory of Microbiology, Department of Biology, Faculty of Life and Natural Sciences, University of Mostaganem, Mostaganem, Algeria

\begin{abstract}
New ternary membranes have been synthesized and characterized. The membranes have been elaborated with natural polymers such as "corn starch" and "Bentonite" added with activated carbon. The technique of phase reversal induced by a solvent at ambient temperature has been applied for the development of these membranes. A set of characterization techniques which include FTIR, SEM are used for structural, morphological knowledge. The ternary membranes are performed on microbiological separation for the filtration of bacteria (Escherichia coli, Pseudomonas aeruginosa) and fungus (Aspergillus niger, Penicillium notatium) and compared to binary membranes which include natural polymers such as "corn starch" and "Bentonite" as compositions. Results showed that the ternary membrane has high performance compared to binary membranes for the filtration Escherichia coli, Pseudomonas aeruginosa and fungi Aspergillus niger.
\end{abstract}

Keywords: Natural polymers; Synthesis, Characterization; Bacteria; Fungi; Separation

\section{Introduction}

Membrane technologies have been widely studied in recent years $[1,2]$. The key efficient and economical separation of this process is the membrane [3], which is defined as a physical barrier to retain or pass microorganisms. The most important desired characteristics of the membranes are the good permeability, high selectivity and stability [3-5]. Today, the membrane separation must now be considered as powerful advanced technologies and convenient to use [6]. Their installation and implementation requires attention and special expertise [7-9]. The application of membrane filtration technology in the field of microbiology requires the development of new membranes based on natural polymers such as corn starch and bentonite [10]. The use of a biopolymer such as starch can be an interesting solution because such a polymer is relatively inexpensive, abundant and biodegradable. The inversion technique of induced phase by solvent has been applied to the preparation of these membranes [11]. This technique is most suitable for our selected polymers $[12,13]$. Our work involves the development of two types of membrane, the binary starch and bentonite membrane and ternary membrane by adding commercial activated carbon to the first. The incorporation of the latter material was a need as there was increased retention of certain microorganisms selected during filtration.

\section{Materials and Methods}

Natural powder of corn starch is extracted from maize raw material. Two types of commercial bentonite sodium were obtained from BENTAL Company "ENOF" of Mostaganem. A commercial activated carbon (REIDEL) was purchased from Maen (Germany) and the distillated water was used as solvent during membranes fabrications. Bacterial strains (Escherichia coli, Pseudomonas aeruginosa) were obtained from laboratory hospital of Mostaganem, and fungi strains (Aspergillus niger, Penicillium sp) were obtained from microbiology laboratory, department of biology of Mostaganem/Algeria.

\section{Membrane preparation}

The membrane preparation technique used in our work requires corn starch, bentonite and commercial activated carbon (REIDEL). A series of membranes with various proportions of these three materials was developed. The technique of induced phase inversion by solvent is used to make an asymmetric support with thin layers and high porosity, this method allows the fabrication of flat asymmetric hybrid membranes at different concentrations of polymers and activated carbon. The selected suitable solvent was distilled water for reasons of cost, safety, availability and its chemical affinity with natural polymers. As the corn starch is insoluble in cold water, the corn starch prepared solutions are heated to a temperature of $70^{\circ} \mathrm{C}$ and stirred magnetically for a period of 90 minutes, after the solubility test of the corn starch, the selected solution in which the concentration is $25 \mathrm{~g} / \mathrm{L}$, this solution is called a starch paste. The incorporation of bentonite and activated carbon to the starch solution allows the preparation of ternary and binary mixture whose protocol is as follows. $20 \mathrm{ml}$ of the prepared corn starch solution was added to different amounts of treated/untreated bentonite and various proportions of activated carbon. The mixture is heated under magnetic stirring for two hours to ensure solution homogeneity. $1 \mathrm{ml}$ were collected from the mixture which then spread on a filter paper type Macherey-Nagel on circular diameter of $7 \mathrm{~cm}$ using a metal roll. The prepared membranes were dried in an oven and maintained at a moderate temperature, the membranes then wrapped in aluminum foil to sterilize those that have been applied to microbiological filtration. The characterization was performed on selected membranes after filtration microbiological tests.

\section{Microbiological separation}

Strains of bacteria such as (Escherichia coli, Pseudomonas aeruginosa) and fungus (Aspergillus niger, Penicillium sp) were activated and purified by Gram staining technique before any microbiological test filtration. All conditions were prepared in the laboratory using conventional microbiological techniques: the broth and nutrient agar for bacteria, broth and Sabouraud agar for fungus. After activation of

*Corresponding author: Hammadi Kheira, Department of Chemistry, Faculty of Sciences and Technology, University of Mostaganem, Mostaganem, Algeria, Tel: 213555114222; E-mail: kyrabiology@yahoo.fr

Received June 12, 2017; Accepted July 03, 2017; Published July 07, 2017

Citation: Bouhadiba K, Djennad MH, Hammadi K (2017) Development and Characterization of Ternary Hybrid Membranes in Natural Polymers and their Application in the Microbiology Field. Mod Chem appl 5: 224. doi: 10.4172/23296798.1000224

Copyright: @ 2017 Bouhadiba K, et al. This is an open-access article distributed under the terms of the Creative Commons Attribution License, which permits unrestricted use, distribution, and reproduction in any medium, provided the original author and source are credited. 
the different strains, the bacterial suspensions and those of fungus were reactivated before each test with respecting of the filtration duration ( 24 to 72 hours) and the incubation temperature $\left(27^{\circ} \mathrm{C}\right.$ to $\left.30^{\circ} \mathrm{C}\right)$. The filtration was carried out in the microbiology laboratory in well sterile conditions to avoid any air contaminations. After the filtration test on wide range of developed membranes, series membranes have been selected in which the bentonite concentration is $100 \mathrm{~g} / \mathrm{L}$ and that of the activated carbon of ternary is $12.5 \mathrm{~g} / \mathrm{L}$. As our goal is to design selective polymeric membranes. The selected series of membranes present the retention property for the selected microorganisms.

\section{Results and Discussion}

\section{Membrane characterization}

The prepared membranes are characterized using two methods, the top surface morphologies of membranes were observed by scanning electron microscopy (Hitachi TM 1000) which can achieve $30000 \mathrm{~nm}$ of resolution. The chemical group an interaction is identified using Fourier Transform Infrared Spectroscopy (FTIR) (SHIMADZU IR Prestige-21), range of wave number is $400-4000 \mathrm{~cm}^{-1}$.

\section{Fourier Transform Infrared Spectroscopy (FTIR)}

Fourier transform infrared spectroscopy was utilized to identify vibration modes of different functional groups of prepared membranes $M_{1}, M_{2}, M_{3}$ and $M_{4}$. The results are shown in Figure 1. As can be observed, the four membranes IR Specters are similar in the range of $4000-1000 \mathrm{~cm}^{-1}$, it seems that the peaks intensity decreases during the addition of activated carbon. The band intensity caused by the stretching vibrations $\mathrm{Si}-\mathrm{O}-\mathrm{Si}$ (1035 -1024-1037-1042) and the bands due to deformation vibrations $\mathrm{Al}-\mathrm{OH}\left(915 \mathrm{~cm}^{-1}\right.$ for the membranes $\mathrm{M}_{1}$ et $\mathrm{M}_{2}$ ) were not affected by the addition of activated carbon as shown in Figures 1-4.

\section{Scanning Electron Microscopy (SEM)}

As shown on Figures 5 and 6 , the photos a, b, c and d corresponding to the membranes $M_{1}, M_{2}, M_{3}$ and $M_{4}$ shows that the surfaces have pores with size and distribution and shape more or less heterogeneous.

\section{Performance evaluation of ternary membranes compared to binary membranes}

The microbiological experiments was used to test the membranes $M_{1}, M_{2}, M_{3}$ and $M_{4}$ the ability of these to filtrate the microorganisms;
Escherichia coli, Pseudomonas aeruginosa as bacterial test and Penicillum notatum and Aspergillus niger as a fungal strains. The microorganisms strains was delivered from the laboratory of microbiology; University of Mostaganem, Algeria, after isolation and preparation of the cultures at specified milieu the nutrient broth milieu for bacteria and sabouraud liquid for fungi. The results of the filtration of selected membranes $M_{1}$, $M_{2}, M_{3}$ and $M_{4}$ are grouped in three (Tables 1-4). The first experiment where the membranes $M_{1}, M_{2}, M_{3}$, and $M_{4}$ have been tested on filtration of Escherichia-coli and Pseudomonas aeruginosa microorganisms, the binary membrane with treated bentonite $M_{1}$ has retained the tow microorganisms, but the results were negatives with not treated membrane $\mathrm{M}_{3}$ due the effect of treated bentonite on structure of the membrane pore diameter. The retaining of Escherichia coli and Pseudomonas aeruginosa bacteria has been enhanced after adding the activated carbon on the membrane $\mathrm{M}_{3}$ for the results of the filtration. Aspergillus niger and Penicillium notatum in Table 4, the membranes $\mathrm{M}_{3}$ have showed a negative results contrary with bacteria showed in Table 3. For the membrane $M_{4}$, the retention of Aspergillus can be explained by the morphology of this fungi which has a significant mycelium that cannot be passing through diameter holes of the membranes $M_{3}$ and $M_{4}$. The negative result of membranes $M_{1}, M_{2}$ may be due to the morphology spores released by the fungi.

\section{Conclusion}

From this work, a ternary membrane $\mathrm{M}_{2}, \mathrm{M}_{4}$ were successfully prepared by inversion technique of induced phase by solvent, the fabricated membrane is shown a porous surface with heterogeneous morphology. The investigated ternary membranes $M_{2}, M_{4}$, the membrane $\mathrm{M}_{4}$ which is the adding of the activated carbon on (Bentonite/corn starch) binary membrane results in enhanced on microbiological separation compared to binary membranes $M_{1}, M_{2}$. The most important characteristics (pore diameter and porous volume) scanning electron microscopy observation showed homogeneous layers and porous without cracking; the same results were showed by Agoudjil et al. in 2014; where they obtained a silica membranes which can be used in Ultrafiltration [14]. We can say this work; is new approach to think about how we can use the "bentonite"; natural polymer "corn starch" and active carbone to prepare polymeric membrane to filtrate the microorganism; furthermore work is on to see permeability of the prepared membrane using other bacteria; fungi and, viruses.

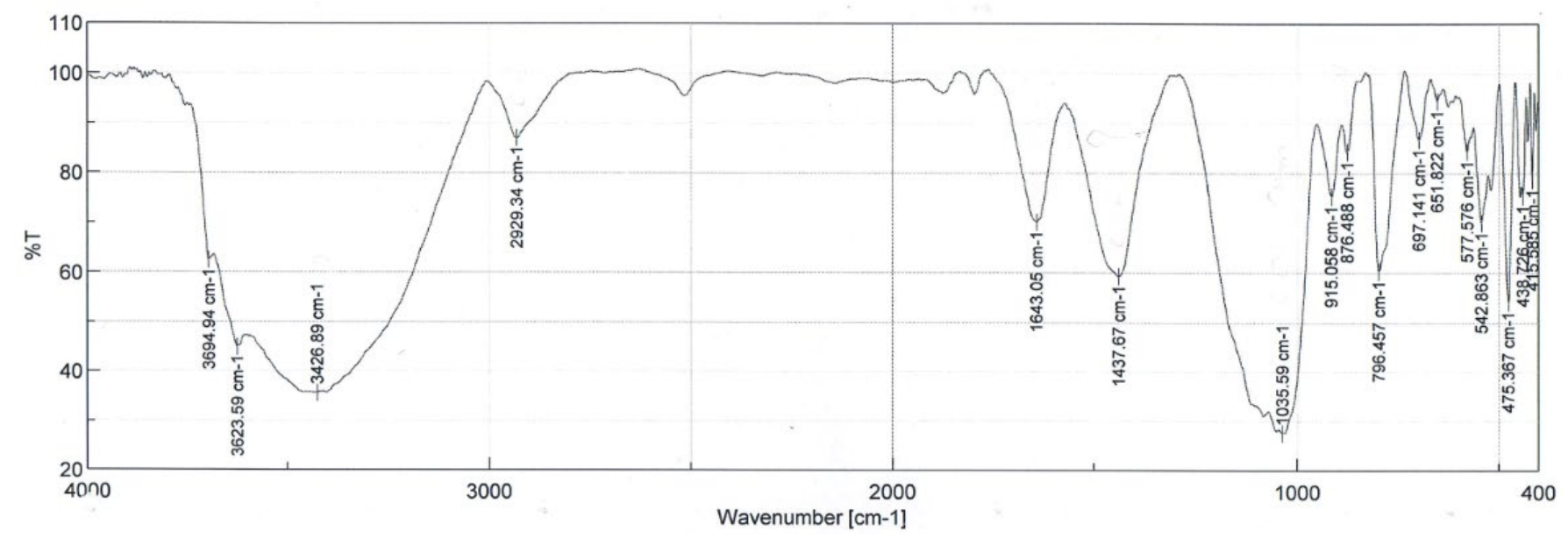

Figure 1: Spectra of top layer of $M_{1}$. 


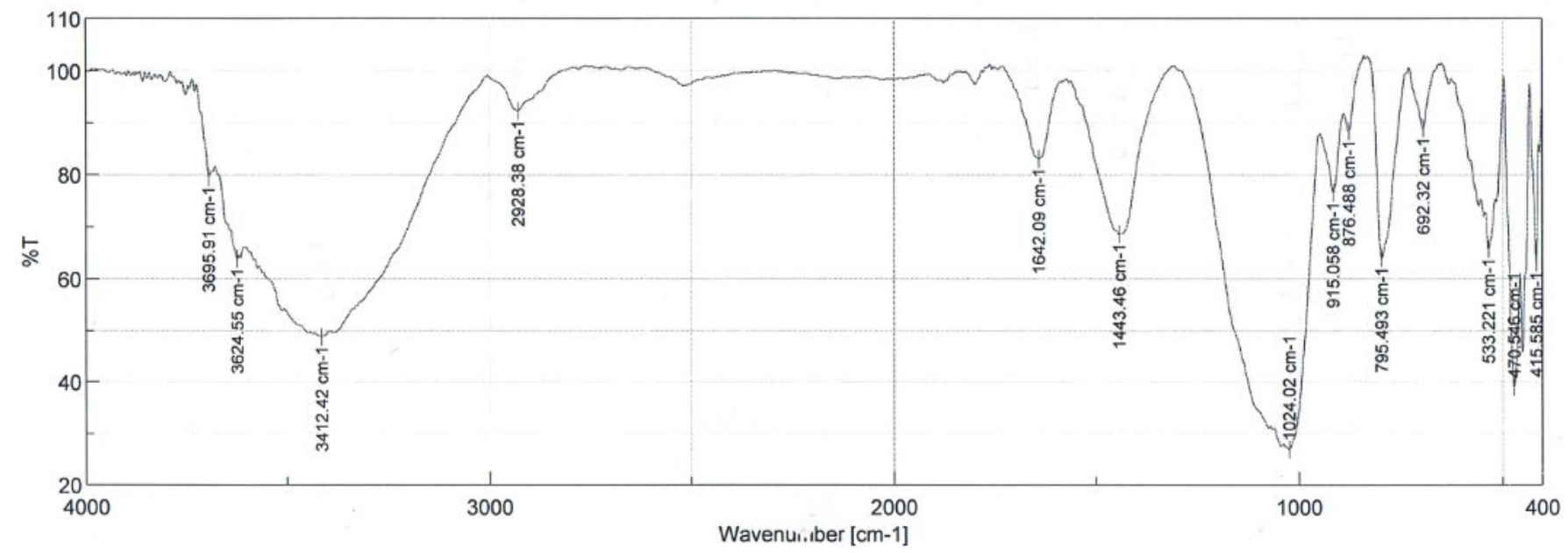

Figure 2: Spectra of top layer of different membrane $M_{2}$.

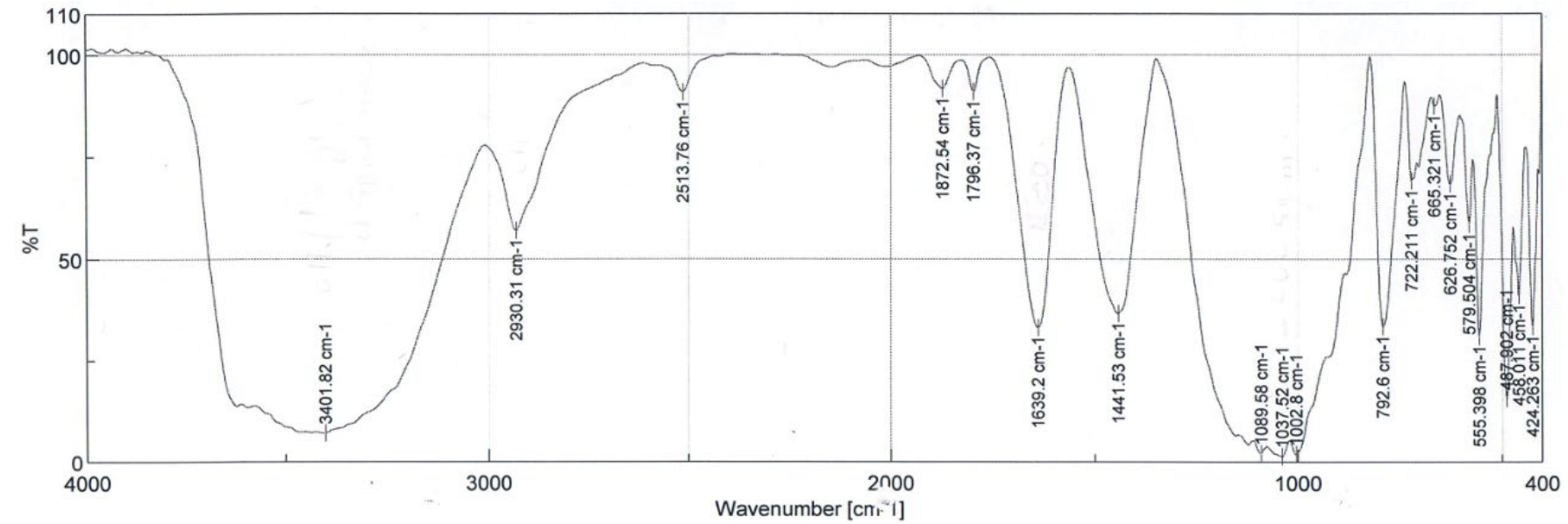

Figure 3: Spectra of top layer of different membrane $M_{3}$.

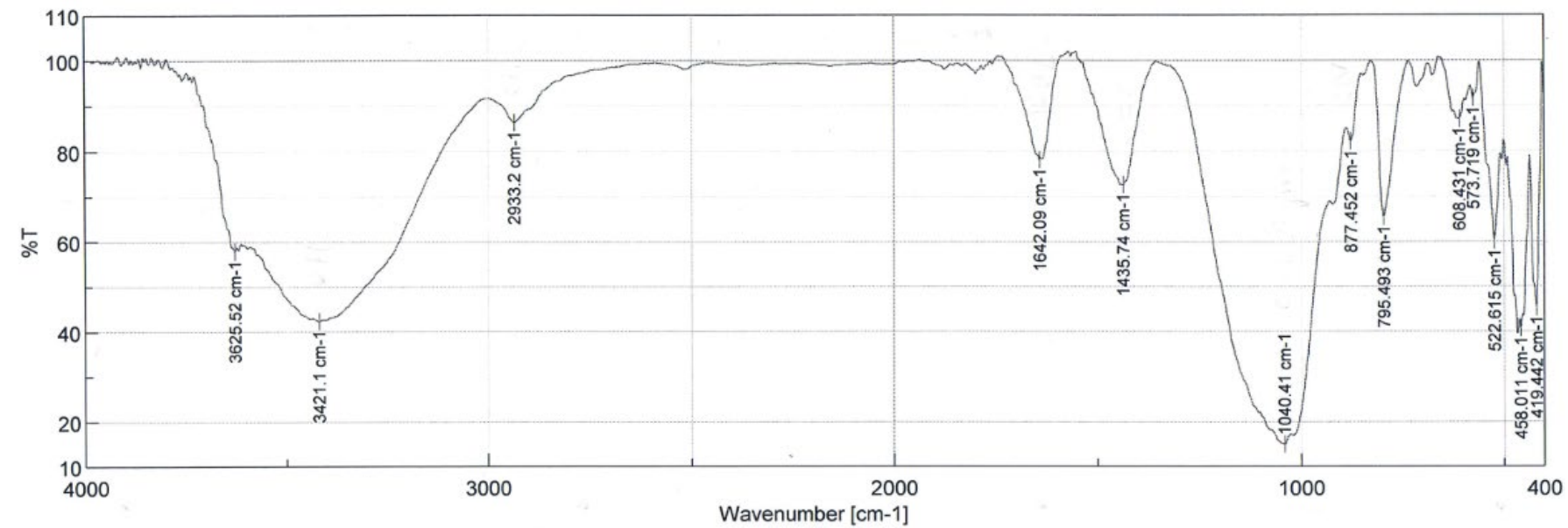

Figure 4: FTIR spectra of top layer of different membrane $M_{4}$. 
Citation: Bouhadiba K, Djennad MH, Hammadi K (2017) Development and Characterization of Ternary Hybrid Membranes in Natural Polymers and their Application in the Microbiology Field. Mod Chem appl 5: 224. doi: 10.4172/2329-6798.1000224

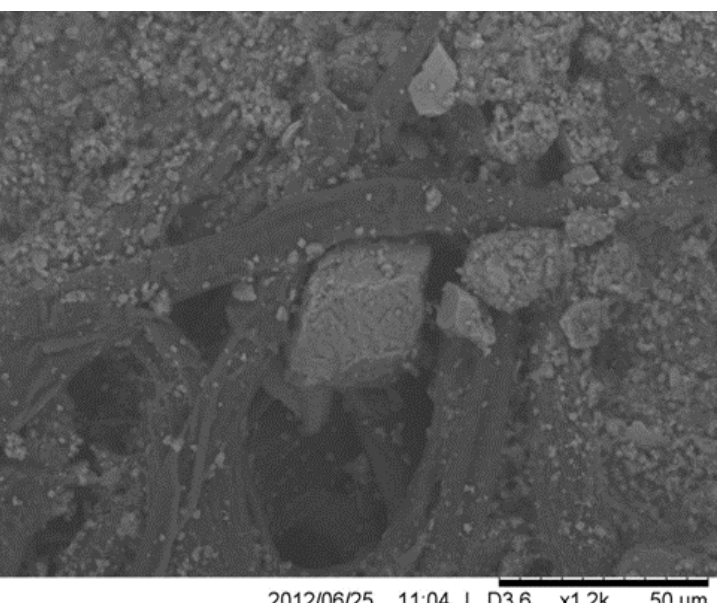

TM1000-0089-06-12

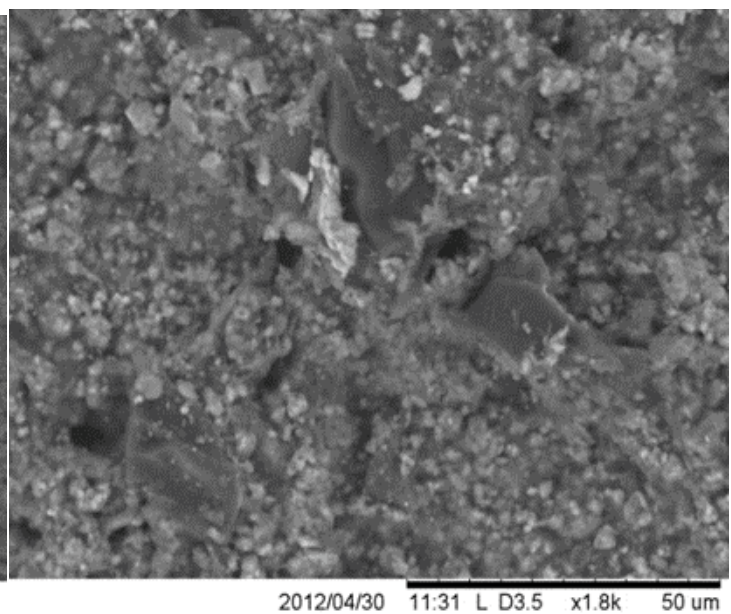

TM1000-0267-04-12

Figure 5: SEM photographs for binary and ternary membranes: (a) $M_{1}$, (b) $M_{2}$.

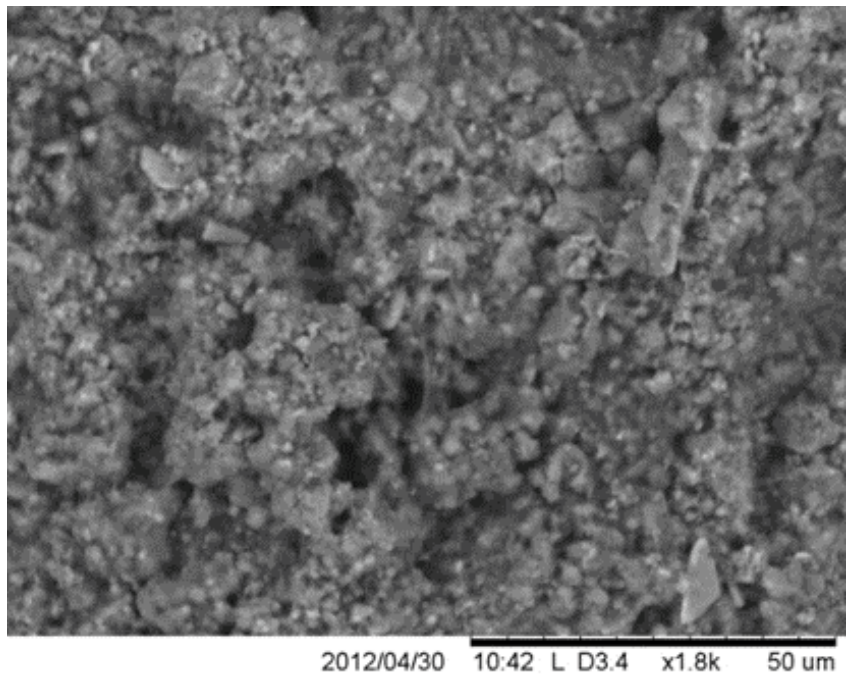

TM1000-0257-04-12

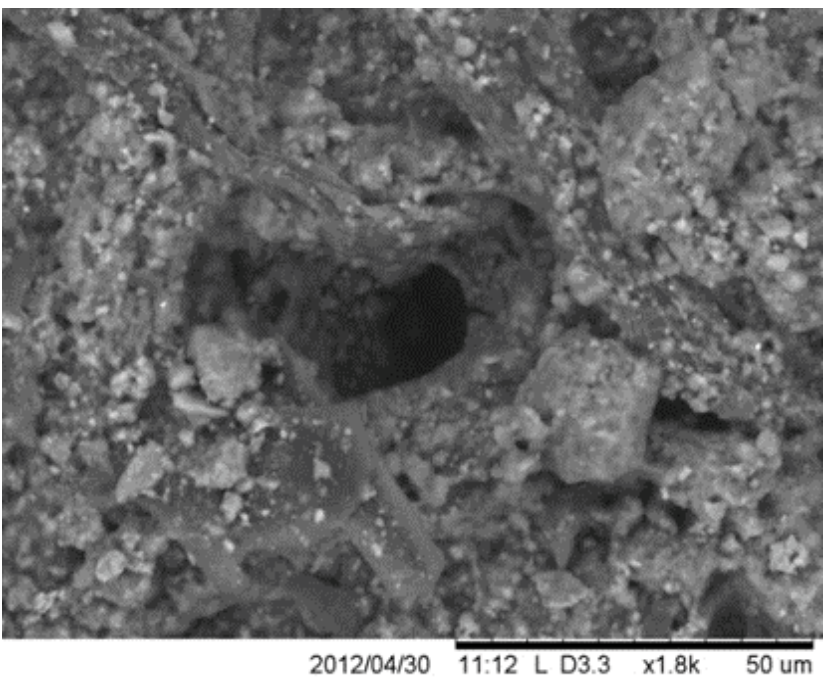

TM1000-0262-04-12

Figure 6: SEM photographs for binary and ternary membranes: (c) $M_{3}$, (d) $M_{4}$.

\begin{tabular}{|c|c|c|c|}
\hline $\begin{array}{c}\text { Selected } \\
\text { Membrane }\end{array}$ & $\begin{array}{c}\text { Amidon } \\
\text { Concentration } \\
\text { (g/L) }\end{array}$ & $\begin{array}{c}\text { Bentonite } \\
\text { concentration (g/L) }\end{array}$ & $\begin{array}{c}\text { Activited carbon } \\
\text { concentration (g/L) }\end{array}$ \\
\hline $\mathrm{M}_{1}$ & 25 & 100 & $/$ \\
\hline $\mathrm{M}_{2}$ & 25 & 100 & 12.5 \\
\hline $\mathrm{M}_{3}$ & 25 & 100 & $/$ \\
\hline $\mathrm{M}_{4}$ & 25 & 100 & 12.5 \\
\hline
\end{tabular}

$M_{1}, M_{2}$ : Synthesized membranes with treated Bentonite; $M_{3}, M_{4}$ : Synthesized membranes with natural Bentonite

Table 1: Different selected membranes.

Bacterial strains membranes $\quad$ Escherichia coli Pseudomonas aeroginosa

\begin{tabular}{|c|c|c|}
\hline$M_{1}$ & - & - \\
\hline$M_{2}$ & + & - \\
\hline$M_{3}$ & + & + \\
\hline$M_{4}$ & - & - \\
\hline
\end{tabular}

$(+)$ Presence of bacteria in the filtrate; (-) Absence of bacteria in the filtrate. Table 2: Results of bacteria filtration.

\begin{tabular}{|c|c|c|}
\hline Fungal strains membranes & Aspergillus niger & Penicillium sp \\
\hline $\mathrm{M}_{1}$ & + & + \\
\hline $\mathrm{M}_{2}$ & + & + \\
\hline $\mathrm{M}_{3}$ & - & - \\
\hline $\mathrm{M}_{4}$ & - & + \\
\hline
\end{tabular}

$(+)$ Presence of bacteria in the filtrate; (-) Absence of bacteria in the filtrate Table 3: Results of the $A$. niger and $P$. notatum filtration.

\begin{tabular}{|c|c|}
\hline Tested microorganisms & Selective membranes \\
\hline Escherichia coli & $\mathrm{M}_{1}, \mathrm{M}_{4}$ \\
\hline Pseudomonas aeruginosa & $\mathrm{M}_{1}, \mathrm{M}_{2}, \mathrm{M}_{4}$ \\
\hline Aspergillus niger & $\mathrm{M}_{3}, \mathrm{M}_{4}$ \\
\hline Penicillium notatum & $\mathrm{M}_{3}$ \\
\hline
\end{tabular}

Table 4: The retained selective membranes.

\section{References}

1. Jean PB (1988) Membrane separation processes. Transport Membrane techniques. Application. Masson, pp: 123-147. 
Citation: Bouhadiba K, Djennad MH, Hammadi K (2017) Development and Characterization of Ternary Hybrid Membranes in Natural Polymers and their Application in the Microbiology Field. Mod Chem appl 5: 224. doi: 10.4172/2329-6798.1000224

2. CFM 2 Booklet (2002) Micro and ultrafiltration: conduction and pilot tests. Treatment of buckets and effluents.

3. Audinos R, Isoard P (1986) Glossary: Technical terms of membrane processes. Société française de filtration, pp: 142

4. Medina GY (2015) Study of solubilization phenomena Pre-polymers of epoxy resin in fatty acid esters as Biosolvents. Doctoral Thesis. INP of Toulouse, France.

5. Bessiere $Y(2005)$ Front membrane filtration: highlighting of the critical filter volume for anticipation and control of clogging, Toulouse. Doctoral Thesis.

6. Hassani L, Pontie M, Dach H, Diawara C (2006) Application of membrane technologies in drinking water production. Interests and limits of use. Mali Symposium on Applied Sciences.

7. Micro and Ultrafiltration (2002) Conduct of pilot tests, treatment of water and effluents.

8. Cheruan M (1998) Ultrafiltration and microfiltration handbook. Technomic Publishing Co. Inc., Lancaster, p: 552
9. Guide to Nano Filtration (2000) CFM Handbook.

10. Maillevialle J, Odendaal PE, Weisner MR (1996) The emergence of Membranes in water and waste water treatment. In: Water Treatment Membrane Process.

11. Drioli E, Criscuoli A, Curcio E (2005) Membrane Contactors: Fundamentals Applications and Potentialities: Membrane Science and Technology. Membrane Science and Technology, p: 516.

12. Kang YS, Kim SH, Young J, Won J (2004) Influence of the addition of PVP on the morphology of asymmetric polyimide phase inversion membranes: Effect of PVP molecular weight. Journal of Membrane Science 236: 203-207.

13. Roosta A, Mousavi S, Ramazani A, Roshan MA (2006) Correlation of Nitrogen Enriching Polymeric Membranes Performance Developed through Various Methods with their Morphological Structure Revealed via SEM. Iranian Polymer Journal 15: 291-298.

14. Agoudjil N, Lamrani A, Larbot L (2014) Silica porous membranes synthesis and characterization Desalination and Water Treatment. Desalination and Water Treatment 15: 2988-2995. 